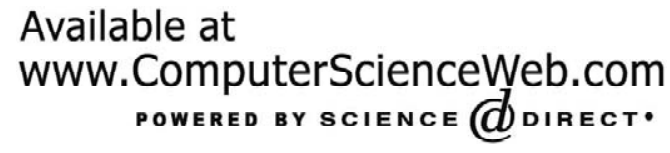

Medical Image Analysis 7 (2003) 489-502 $\overline{\overline{\text { MEDICAL }}}$ IMAGE

ANALYSIS

www.elsevier.com/locate/media

\title{
From colour to tissue histology: Physics-based interpretation of images of pigmented skin lesions
}

\author{
Ela Claridge ${ }^{\mathrm{a}, *}$, Symon Cotton $^{\mathrm{b}}$, Per Hall ${ }^{\mathrm{c}}$, Marc Moncrieff ${ }^{\mathrm{d}}$ \\ ${ }^{a}$ School of Computer Science, The University of Birmingham, Birmingham B15 2TT, UK \\ ${ }^{\mathrm{b}}$ Astron Clinica, The Mount, Toft, Cambridge CB3 7RL, UK \\ 'Department of Plastic Surgery, Addenbrooke's Hospital, Cambridge CB2 2QQ, UK \\ ${ }^{\mathrm{d}}$ Mersey Deanery, Hamilton House, 24 Pall Mall, Liverpool L3, UK
}

\begin{abstract}
Through an understanding of the image formation process, diagnostically important facts about the internal structure and composition of pigmented skin lesions can be derived from their colour images. A physics-based model of tissue colouration provides a cross-reference between image colours and the underlying histological parameters. It is constructed by computing the spectral composition of light remitted from the skin given parameters specifying its structure and optical properties. The model is representative of all the normal human skin colours, irrespective of racial origin, age or gender. Abnormal skin colours do not conform to this model and thus can be detected. Once the model is constructed, for each pixel in a colour image its histological parameters are computed from the model. Represented as images, these 'parametric maps' show the concentration of dermal and epidermal melanin, blood and collagen thickness across the imaged skin as well as locations where abnormal colouration exists. In a clinical study the parametric maps were used by a clinician to detect the presence of malignant melanoma in a set of 348 pigmented lesions imaged using a commercial device, the SIAscope. Logistic regression identified the presence of melanin in the dermis, the abnormal distribution of blood within the lesion and the lesion size as the most diagnostically informative features. Classification based on these features showed $80.1 \%$ sensitivity and $82.7 \%$ specificity in melanoma detection.
\end{abstract}

(c) 2003 Elsevier B.V. All rights reserved.

Keywords: Colour analysis; Image analysis; Physics-based modelling; Pigmented skin lesions; Melanoma diagnosis

\section{Introduction}

Colour is an important sign in the clinical diagnosis of many conditions. In the computer analysis of medical images colour also plays a considerable role, for example in segmentation and classification. These and similar operations utilise colour as one of the image features, but the question "Why is a particular colour associated with a particular medical condition?" is not frequently asked.

How do the colours that we see on the surface arise? Light emitted by a source interacts with the surface and the interior of an object and through these interactions (mainly absorption and scatter) the spectral composition of the light

\footnotetext{
*Corresponding author. Tel.: +44-121-414-4778; fax: +44-121-1414281 .

E-mail address: e.claridge@cs.bham.ac.uk (E. Claridge).
}

is altered. The changes reflect the structure and optical properties of the materials constituting the object and in this sense the light remitted from the object 'encodes' its properties. If this encoding is understood, it should be possible to deduce the structure and composition of the object from its colour image.

In this paper we show how the understanding of the image formation process enables us to derive diagnostically important facts about the internal structure and composition of skin lesions from their colour ${ }^{1}$ images. This information is then used for the clinical diagnosis of pigmented skin lesions to aid the detection of melanoma, a skin cancer.

\footnotetext{
${ }^{1}$ In this the paper 'colour' is taken to be a vector $n$ primaries. For example, a standard colour image is represented by 3 primaries red, green

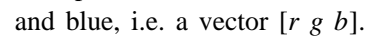




\subsection{Outline of the method}

The key to the interpretation of skin image colours in terms of the underlying histological parameters is a model of tissue colouration which provides a cross-reference between the colour and the histology. This model is constructed by computing the spectra remitted from the skin given parameters specifying its structure and optical properties, and then computing equivalent RGB colours. This step needs to be carried out only once. As the mapping between the colours and the parameters is unique for the skin (Claridge and Preece, 2003) each colour corresponds to one specific set of histological parameters. For each derived parameter a parametric map is then created which shows the magnitude of a given parameter at each pixel location. As an example, Fig. 6 shows an advanced melanoma and a set of histological parametric maps showing the concentration of dermal and epidermal melanin, blood and collagen thickness across the imaged lesion and its surrounding skin. Detailed description and interpretation of the maps is given in Section 5.3.

The new methodology described in this paper can be viewed as a special case of supervised classification in a feature space. The construction of the model of tissue colouration is the training phase, where training is carried out not on 'real' colours found in lesion images but on colours predicted by a model of light transport (see Section 4). A feature space is defined by the histological parameters that the method seeks to recover. In the classification phase, for a given colour in a real image its closest match is found in the feature space thus effectively classifying the colour in terms of its histological parameters. The use of a predictive model instead of real data in the training phase is one of the main differences between this and other colour analysis methods. A full discussion of this method in the context of other relevant research can be found in Section 8 .

\subsection{Structure of the paper}

The paper begins with a review of research on colour analysis of the skin and pigmented skin lesions. Next, the paper describes the structure and optical properties of both the normal and abnormal skin and discusses how they affect the composition of light remitted from the skin. The method for constructing a model of colouration for the normal skin is then described, including details of the model of light transport based on Kubelka-Munk theory. The next section describes the results, including the parametric maps depicting the changes in skin structure associated with early and advanced melanoma. Section 6 describes experiments carried out to validate the model. Section 7 presents the results of clinical evaluation, followed by discussion and conclusions.

\section{Colour analysis of the skin and pigmented skin lesions}

The importance of colour in lesion diagnosis is reflected by the fact that colour is included in the two commonly used clinical checklists for pigmented skin lesions, the Seven Point checklist (MacKie, 1985) and the American ABCD list (Fitzpatrick et al., 1988). Both lists compute scores based on change in lesion size, shape, colour, the character of the border and elevation. Dark, bluish or variegated colours are deemed suspicious. Colour also features prominently on a checklist for dermatoscopy (also known as epiluminescence microscopy, ELM). With this technique the lesion is examined using a $10 \times$ magnification lens placed directly against skin to which immersion oil has been applied, to remove scatter of light on the air-skin boundary. This has the effect of making the top skin layer semi-translucent thus allowing better inspection of deeper skin layers. Colour features used for lesion scoring include blue-white veil, red-blue areas, multiple colouration, brown dots and globules, blue/gray dots, and many more (Menzies et al., 1996).

Due to its diagnostic importance the analysis of lesion colouration has been undertaken by many research groups. One of the most common approaches is to derive basic statistics of colour primaries in RGB or other colour spaces, for example $\mathrm{L}^{*} \mathrm{u}^{*} \mathrm{v}^{*}$ (Huang, 1999) and HIS (Ganster et al., 2001). Mean, standard deviation and other simple measures are computed for colours occurring in the lesion and in the surrounding skin, providing colour-related quantitative parameters for subsequent lesion classification (Ercal et al., 1994; Bono et al., 1996; Aitken et al., 1996; Ganster et al., 1997; Husemann et al., 1997; Menzies et al., 1997; Seidenari et al., 1999; Kahofer et al., 2002). Instead of mean and standard deviation some methods characterise colour distribution by a whole histogram or as a mixture of Gaussians (e.g. Jones and Regh, 2002). Similar colour features have also been used for lesion segmentation (see a special issue of Computerized Medical Imaging and Graphics, 1992).

In non-medical applications one of the active areas of colour research has been the detection of human skin and segmentation of areas of exposed skin such as the face or hands in web images. This research has made contributions to understanding of the nature of the skin colouration because it had to deal with the problem of unknown illumination. This contrasts with medical imaging, where there is usually some control over lighting conditions. Under arbitrary lighting pixel colours depend on both the skin colours and the incident light and simple statistical methods do not work well (Greenspan et al., 2001). New approaches had to be developed and the most successful have been those which model skin colouration either statistically (e.g. Cai and Goshtasby, 1999) or using physics-based models (e.g. Tsumura et al., 1999, 2001). 


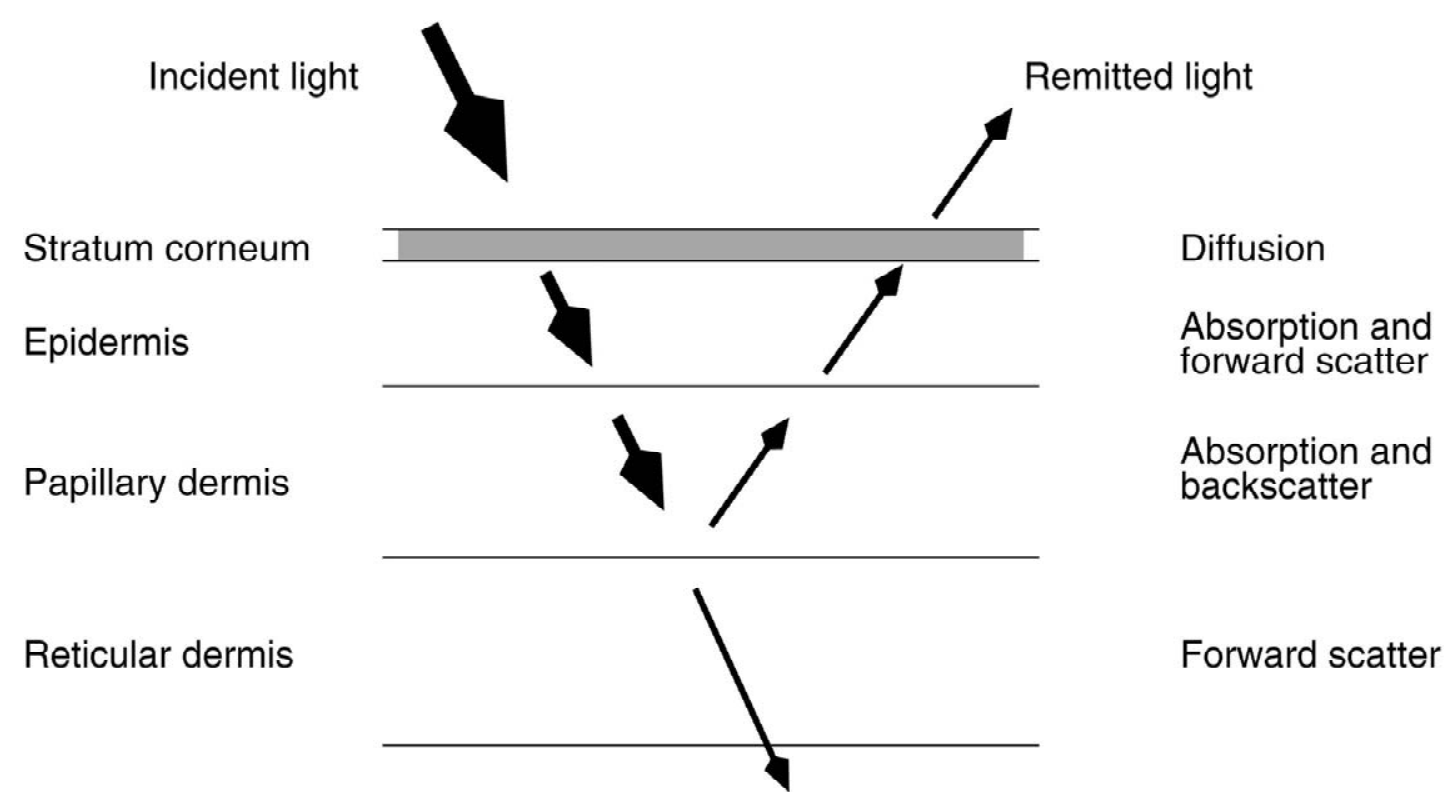

Fig. 1. A schematic representation of the skin layers (names on the left) and their optical properties (on the right). The arrows indicate the path of light through the skin tissues.

In spectral and hyper-spectral analysis, colour is represented by a vector of reflectances at a number of different wavelengths. It is a richer representation than a common three-valued RGB vector. Several groups have used this kind of analysis for lesion classification, typically deriving features characterising the shape of the spectra prior to statistical analysis (e.g. Wallace et al., 2000; Elbaum et al., 2001).

\section{Background}

\subsection{Structure and optical properties of the normal skin}

The skin consists of a number of layers with distinct function and distinct optical properties (Fig. 1). White light ${ }^{2}$ shone onto the skin penetrates superficial skin layers and whilst some of it is absorbed, much is remitted back and can be registered by a camera.

The stratum corneum is a protective layer consisting of keratin-impregnated cells and it varies considerably in thickness. Apart from scattering the light, it is optically neutral.

The epidermis is largely composed of connective tissue. It also contains the melanin producing cells, the melanocytes, and their product, melanin. Melanin is a pigment which strongly absorbs light in the blue part of the

\footnotetext{
'2White' light is assumed to contain all the wavelengths in the visible range in equal proportion.
}

visible spectrum and in the ultraviolet (see Fig. 2). In this way it acts as a filter which protects the deeper layers of the skin from harmful effects of UV radiation. Within the epidermal layer there is very little scattering, with the small amount that occurs being forward directed. The result is that all light not absorbed by melanin can be considered to pass into the dermis.

The dermis is made of collagen fibres and, in contrast to

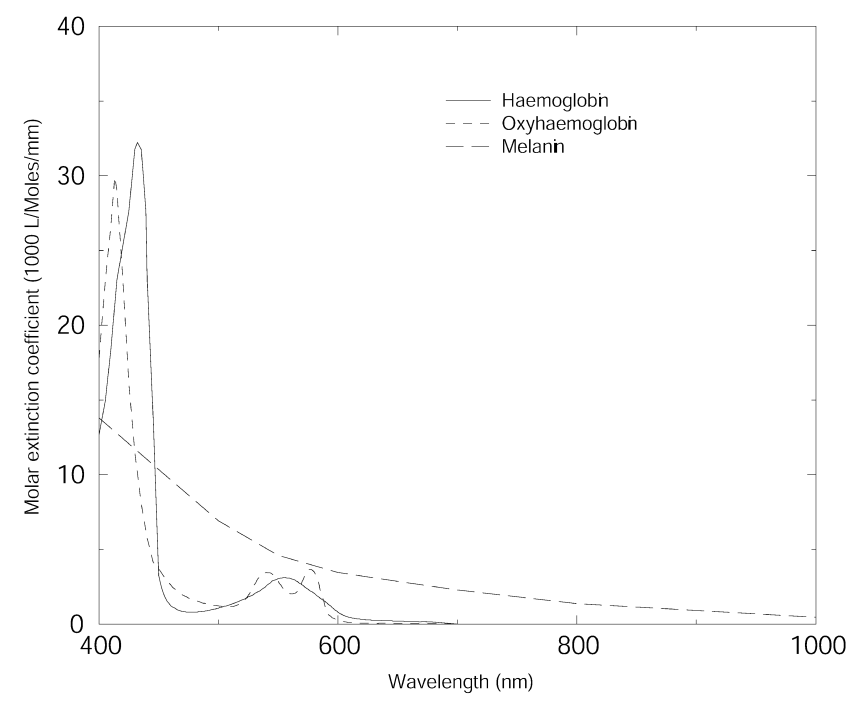

Fig. 2. The absorption spectra for melanin and haemoglobin. In the model a mixture of $50 \%$ haemoglobin and $50 \%$ oxyhaemoglobin have been used. 
the epidermis, it contains sensors, receptors, blood vessels and nerve ends. Haemoglobin, present in blood vessels across the whole dermis, acts as a selective absorber of light (see Fig. 2). The dermis consists of two structurally different layers, papillary and reticular, which differ principally by the size of collagen fibres. The small size of the collagen fibres in the papillary dermis (diameter of an order of magnitude less than the incident visible light) makes this layer highly back-scattering; i.e. any incoming light is directed back towards the skin surface. The scatter is greatest at the red end of the spectrum and increases even further in near infrared (nir). As absorption by melanin and blood is negligible in the infrared, this part of the spectrum is optimal for assessing the thickness of the papillary dermis.

Within the reticular dermis, the large size of collagen fibre bundles causes highly forward-directed scattering. Thus any light which gets to this layer is passed on deeper into the skin and does not contribute to the spectrum remitted from the skin (Fig. 2).

It is important to note that these descriptions characterise all instances of the normal skin. Differences related to a racial type, age or gender are all in terms of the quantities of pigments and thicknesses of various layers.

\subsection{Skin structure associated with pigmented skin lesions}

Pigmented skin lesions appear as patches of darker colour on the skin. In most cases the cause is excessive melanin concentration in the skin. In benign lesions (e.g. common naevi) melanin deposits are normally found in the epidermis. Dermal melanin can be found also in benign lesions (e.g. junctional naevus and blue naevus). Apparent excessive pigmentation can also occur as the result of the papillary dermis becoming thin. In this condition light is absorbed by structures beyond the reticular dermis instead of being back-scattered and hence a smaller fraction is remitted. Sometimes small deposits of blood or large concentrations of small blood vessels can take the appearance similar to a pigmented skin lesion. All these types of lesions have the same structure as the normal skin, the only difference being relatively high levels of pigment concentration or decreased thickness of the papillary dermis.

Malignant melanoma is a skin cancer. It occurs when melanocytes reproduce at a high, abnormal rate. Whilst they and their associated melanin remain in the epidermis, melanoma is termed 'in situ'. At this stage it is not life-threatening and its optical properties make it conform to those of the normal, highly pigmented skin. When malignant melanocytes have penetrated into the dermis, they leave melanin deposits there, changing the nature of skin colouration. The likelihood of metastases increases with the depth of penetration and patient prognosis be- comes progressively worse. According to Breslow (1970), $98 \%$ of lesions with penetration smaller than $0.76 \mathrm{~mm}$ would not recur for at least 5 years after surgery, which is considered to be a good prognosis. Early detection is crucial, as once metastases have entered the blood and lymphatic system, this rapidly growing cancer can effect life threatening damage in vital organs.

The presence of melanin in the dermis is the most significant sign of melanoma. However, it cannot be used as a sole diagnostic criterion because in situ melanomas do not have dermal melanin. Moreover, some benign naevi have dermal deposits, although their spatial patterns tend to be more regular than in melanoma. Other signs, some of which can be indicative of melanoma in situ, are thickening of the collagen fibres in the papillary dermis (fibrosis); increased blood supply at the lesion periphery (erythematous reaction); and lack of blood within the lesion, in the areas destroyed by cancer.

The colours associated with skin which has melanin deposits in the dermis normally show characteristic hues not found in any other skin conditions. This provides an important diagnostic cue for a clinician. However, similar hues also arise in other circumstances, for example for particular concentrations of epidermal melanin in the presence of thinned papillary dermis. This phenomenon is called metamerism and is one of the causes of difficulty in clinical diagnosis.

\section{Developing a model of skin colouration}

As stated in the outline of the method (Section 1.1), prior to image interpretation a model of skin colouration needs to be constructed. This section describes in detail: the model of light transport used to predict spectra associated with specific histological parameters; the relevant facts about optical properties of skin tissue components; the method for computing skin colours; and the parameters used in the computation.

\subsection{Model of light transport}

A model of light transport is a realisation of a particular physics theory of light interaction with the matter. The optical characteristics of the skin tissue are such that a number of different light transport models can be used. This work implemented a two-flux model (Cotton et al, 1997) based on Kubelka-Munk theory (Egan and Hilgeman, 1979). It computes the remitted $(R)$ and transmitted $(T)$ light separately for each layer $i: R_{i}$ and $T_{i}$.

$$
\begin{aligned}
& R_{i}=\frac{\left(1-\beta^{2}\right)\left(\mathrm{e}^{K d}-e^{-K d}\right)}{(1+\beta)^{2} \mathrm{e}^{K d}-(1-\beta)^{2} \mathrm{e}^{-K d}}, \\
& T_{i}=\frac{4 \beta}{(1+\beta)^{2} \mathrm{e}^{K d}-(1-\beta)^{2} \mathrm{e}^{-K d}},
\end{aligned}
$$


where

$K=\sqrt{k(k+2 s)}, \quad \beta=\sqrt{\frac{k}{k+2 s}}$,

$d$ is a layer thickness, $k \propto \mu_{\mathrm{a}}$ and $s \propto \mu_{\mathrm{s}}$.

For an $n$ layered system, values for $R_{12 \ldots n}$ and $T_{12 \ldots n}$ are computed recursively (Spiegel, 1962):

$R_{12 \ldots n}=R_{12 \ldots n-1}+\frac{T_{12 \ldots n-1}^{2} R_{n}}{1-R_{12 \ldots n-1} R_{n-1}}$

and

$T_{12 \ldots n}=\frac{T_{12 \ldots n-1} T_{n}}{1-R_{12 \ldots n-1} R_{n}}$.

The model requires that the incident light is diffuse. This condition is fulfilled because, as can be recalled from Section 3.1, light is diffused as it passes through stratum corneum.

\subsection{Model of the normal skin structure}

From the description of the skin structure in 3.1 , the normal skin can be optically modelled as consisting of three layers:

- epidermis, characterised by the wavelength $(\lambda)$ dependent set of absorption coefficients for melanin, $\mu_{\mathrm{a}}^{m}(\lambda)$, and the melanin concentration, $c^{m}$;

- papillary dermis, characterised by the absorption coefficients for haemoglobin, $\mu_{\mathrm{a}}^{h}(\lambda)$, the haemoglobin concentration, $c^{h}$, the scatter coefficient for collagen, $\mu_{\mathrm{s}}^{p d}$, and the thickness of the collagen layer, $d^{p d}$;

- reticular dermis, characterised by the scatter coefficient, $\mu_{\mathrm{s}}^{r d}$, and the layer thickness, $d^{r d}$.

Skin with dermal melanin deposits does not conform to this structure and thus is not represented by this model.

\subsection{A method for computing skin colours}

By supplying the above parameters and the spectral composition of the incident light $E(\lambda)$ to a model of light transport, the spectral composition of the light remitted from the skin, $R(\lambda)$, can be computed:

$R(\lambda)=$ Model_of_light_transport $\left(E(\lambda), \mu_{\mathrm{a}}^{m}(\lambda), c^{m}\right.$,

$\left.\mu_{\mathrm{a}}^{h}(\lambda), c^{h}, \mu_{\mathrm{s}}^{p d}, d^{p d}, \mu_{\mathrm{s}}^{r d}, d^{r d}\right)$.

In the final step a colour vector $[r g b$ nir], is derived from the remitted spectrum $R(\lambda)$ by convolving it with suitable spectral response functions for the red, green, blue and near infrared primaries, $S_{R}(\lambda), S_{G}(\lambda), S_{B}(\lambda)$ and $S_{N I R}(\lambda)$ :

$r=\int_{0}^{\infty} R(\lambda) S_{R}(\lambda) \mathrm{d} \lambda, \quad g=\int_{0}^{\infty} R(\lambda) S_{G}(\lambda) \mathrm{d} \lambda$,
$b=\int_{0}^{\infty} R(\lambda) S_{B}(\lambda) \mathrm{d} \lambda, \quad$ nir $=\int_{0}^{\infty} R(\lambda) S_{\mathrm{NIR}}(\lambda) \mathrm{d} \lambda$.

The process of building of the model of colouration can be described by the following fragment of a pseudocode.

given

incident light $E(\lambda)$

absorption coefficients of melanin and blood, $\mu_{\mathrm{a}}^{m}(\lambda)$, $\mu_{\mathrm{a}}^{h}(\lambda)$

scatter coefficient of the papillary dermis, $\mu_{\mathrm{s}}^{p d}$

scatter coefficient and thickness of the reticular dermis, $\mu_{\mathrm{s}}^{r d}, d^{r d}$

spectral response functions for the red, green blue and nir primaries,

$S_{R}(\lambda), S_{G}(\lambda), S_{B}(\lambda)$ and $S_{\mathrm{NIR}}(\lambda)$

for all valid concentrations of epidermal melanin, $c^{m}$

for all valid concentrations of dermal blood, $c^{h}$

for all valid thicknesses of papillary dermis, $d^{p d}$ compute

$R(\lambda)=$ Model_of light_transport $\left(E(\lambda), \mu_{\mathrm{a}}^{m}(\lambda)\right.$, $\left.c^{m}, \mu_{\mathrm{a}}^{h}(\lambda), c^{\bar{h}}, \bar{\mu}_{\mathrm{s}}^{p d}, \bar{d}^{p d}, \mu_{\mathrm{s}}^{r d}, d^{r d}\right)$

colour vector $\left[\begin{array}{llll}r & g & b & n i r\end{array}\right]$

where

$$
\begin{aligned}
& r=\int_{0}^{\infty} R(\lambda) S_{R}(\lambda) \mathrm{d} \lambda, \\
& g=\int_{0}^{\infty} R(\lambda) S_{G}(\lambda) \mathrm{d} \lambda, \\
& b=\int_{0}^{\infty} R(\lambda) S_{B}(\lambda) \mathrm{d} \lambda, \\
& \text { nir }=\int_{0}^{\infty} R(\lambda) S_{\mathrm{NIR}}(\lambda) \mathrm{d} \lambda .
\end{aligned}
$$

This forward process computes explicitly tissue colour given a set of histological parameters. As the mapping between the parameters and the primaries is unique for the skin (Claridge and Preece, 2003), the inverse mapping is possible: from the tissue colour to its histological parameters:

$[r g$ bir $] \leftrightarrow\left[c^{m} c^{h} d^{p d}\right]$

The quantities $\left[c^{m} c^{h} d^{p d}\right]$ are then used to construct parametric maps.

\subsection{Parameters used in the method}

Parameters in the Model_of_light_transport( ) above can be subdivided into those which characterise the entire tissue type and those which characterise a specific instance of the tissue. The absorption and scatter coefficients $\left(\mu_{\mathrm{a}}^{m}(\lambda), \mu_{\mathrm{a}}^{h}(\lambda), \mu_{\mathrm{s}}^{p d}, \mu_{\mathrm{s}}^{r d}\right)$ belong to the first group and their specific values are based on published data (e.g. 
Table 1

Values and ranges of coefficients used to construct the model of colouration for the normal skin

\begin{tabular}{llll}
\hline Parameter & Symbol & $\begin{array}{l}\text { Range } \\
\text { from }\end{array}$ & $\begin{array}{l}\text { Range } \\
\text { to }\end{array}$ \\
\hline $\begin{array}{l}\text { Epidermal melanin concentration } \\
\left(10^{-1} \mathrm{mmol} / \mathrm{l}\right)\end{array}$ & $c^{m}$ & 0.00 & 8.69 \\
$\begin{array}{l}\text { Haemoglobin concentration } \\
\text { (in dermal blood) (mmol/1) }\end{array}$ & $c^{h}$ & 0.00 & 4.34 \\
$\begin{array}{l}\text { Thickness of the papillary dermis } \\
\left(10^{-4} \mathrm{~m}\right)\end{array}$ & $d^{p d}$ & 0.05 & 0.40 \\
\hline
\end{tabular}

Anderson et al., 1981). Fig. 2 shows the absorption spectra for melanin and haemoglobin. The thickness of the reticular dermis can be assumed constant because due to its strong forward scattering properties even a thin layer will prevent any remission of light.

The levels of melanin and blood concentration $c^{m}$ and $c^{h}$, and thickness of the papillary dermis, $d^{p d}$, vary both between individuals and for different skin locations. The model captures this variability by computing a set of colour vectors for parameters spanning the entire range of histologically valid concentrations and thicknesses. In this way a cross-reference between histology and colour is formed. Table 1 lists the parameter ranges used in this work.

The range of wavelengths used for computing the remitted spectra, from $\lambda=400$ to $1300 \mathrm{~nm}$, covers the

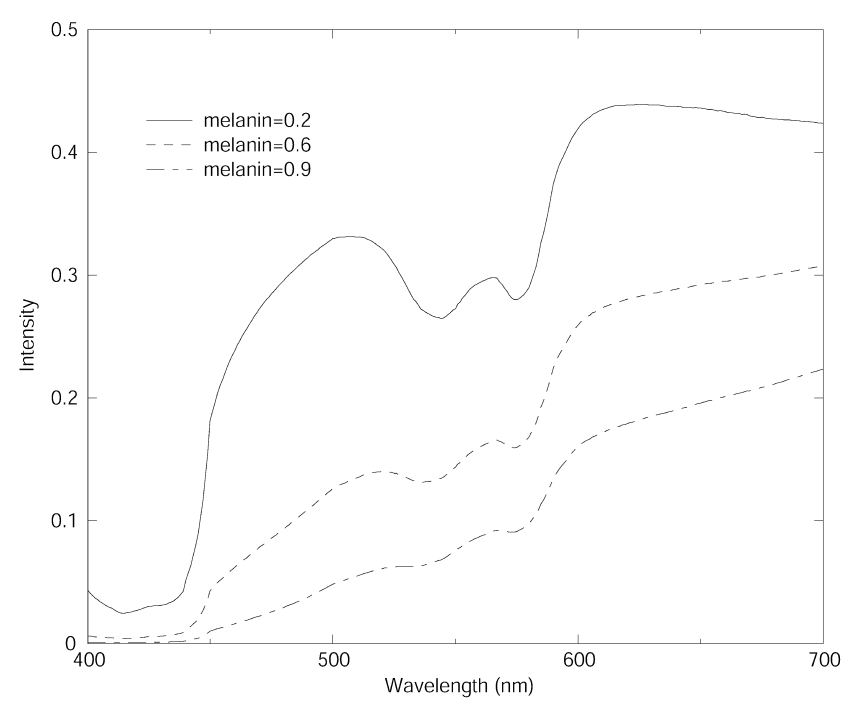

Fig. 3. Examples of reflectance spectra for the normal skin computed using Kubelka-Munk theory. The three curves correspond to three different melanin contents of the skin, with levels of blood and collagen thickness being equal and set to the average values. Two main effects of the melanin increase are the overall drop in the level of remitted light, greater in the red region of the spectrum; and the flattening of the 'double dip', characteristic of haemoglobin, in the green region of the spectrum. whole visible spectrum and a small range of near infrared radiation. The wavelengths used for computations are taken at equal intervals of $30 \mathrm{~nm}$, giving 30 discrete points for each spectrum. The incident light is white, i.e. it has equal contributions from each discrete wavelength. The

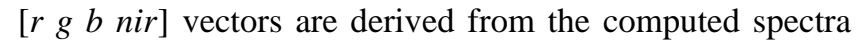
using a set of response functions equivalent to physical filters used by a specific camera.

\section{Results}

\subsection{Model outputs for the normal skin}

Fig. 3 shows three examples of the spectra generated for the constant concentration of haemoglobin, constant thickness of papillary dermis and varying levels of melanin concentration.

The spectra associated with all possible combinations of parameter values are convolved with R, G, B and NIR filters, thus generating a set of image colours. Fig. 4 represents schematically the relationship between the two reference systems: colour, $[r g b]$; and histological parameters: melanin concentration, blood concentration and thickness of the papillary dermis, $\left[c^{m} c^{h} d^{p d}\right]$. These relationships constitute the model of skin colouration. Since there is a one-to-one mapping between the colours and the parameters, the parameter values can be retrieved from the model given the colour vector obtained from each point in a colour image of the skin. The magnitude of each parameter is then displayed at each pixel location in a separate image, giving three parametric maps: melanin, haemoglobin and papillary dermis.

\subsection{Abnormal skin}

The colours of the skin with melanin deposits in the dermis no longer fit the model of normal colouration. In the schematic representation shown in Fig. 4 such colours appear below a 'surface' of a particular papillary dermis thickness. This non-conformance to the model identifies them as being 'abnormal' and provides a highly sensitive diagnostic sign. The change in colouration is relatively large even for a very small depth of penetration. Fig. 5 illustrates the behaviour of the blue primary with the increase of depth at which melanin is found. The initial change is quite rapid and then flattens off as the depth increases. It should be stressed that the presence of dermal melanin can be detected at any depth, but the accuracy of measurement decreases with increase in depth and increase in melanin concentration. It can be seen that even at a very small depth of penetration there will be a significant difference between value of the blue primary for no dermal 


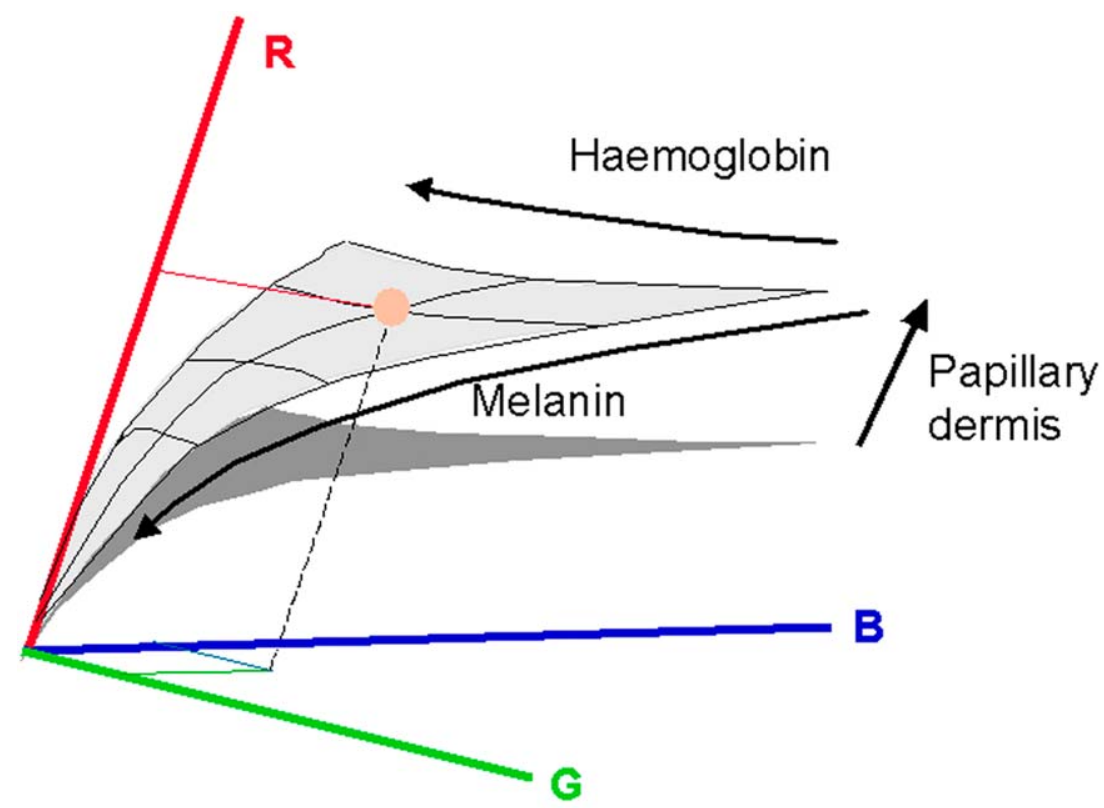

Fig. 4. Schematic relationship between two reference systems: colour system, with axes R, G and B; and histological parameter system, with axes Haemoglobin, Melanin and Papillary Dermis. (This figure is available in colour, see the on-line version).

melanin and melanin at that depth. Similar behaviour is shown by other primaries. Recalling that the threshold Breslow thickness is $0.76 \mathrm{~mm}$, the ability to detect dermal melanin at even smaller depth makes this method very effective in identifying early melanomas and thus contributing to positive prognosis for the patients.

\subsection{Parametric maps}

For each lesion four parametric maps are computed. Fig. 6 shows an image of an advanced melanoma and its parametric maps. First of all the presence of dermal melanin should be noted. The pattern is irregular and

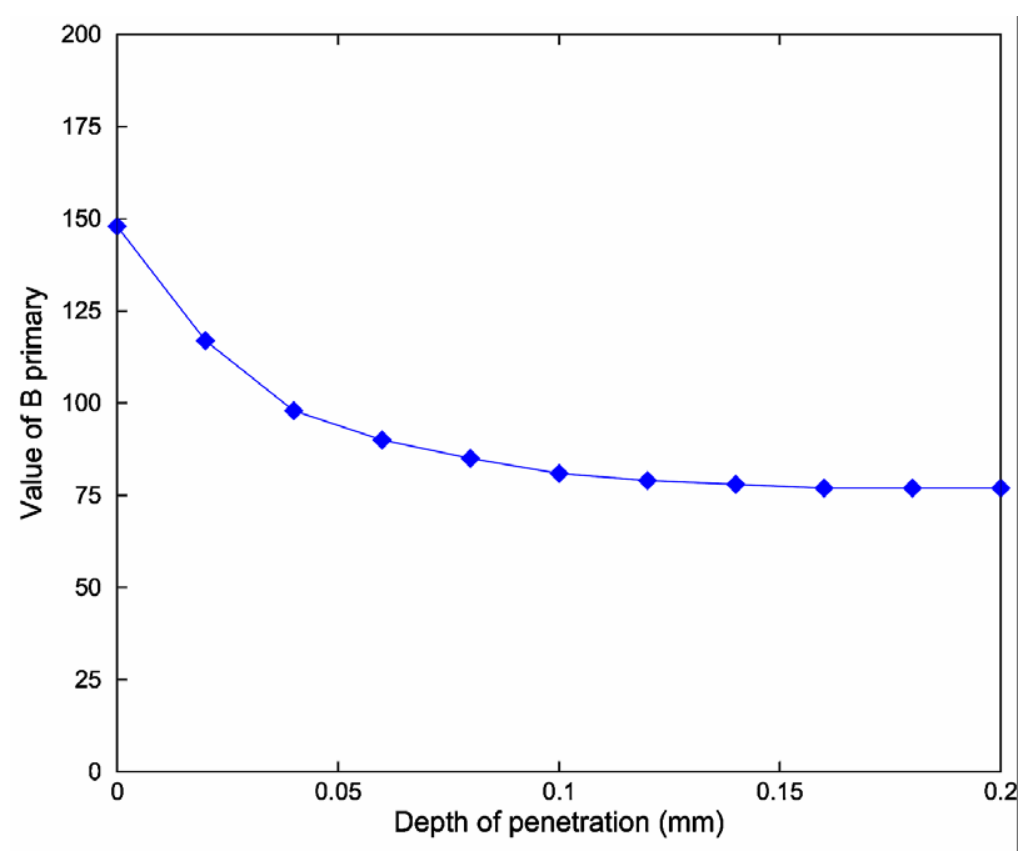

Fig. 5. The behaviour of the blue primary with the increase in depth of dermal melanin. A vertical bar shows a typical standard error of a CCD camera. It can be seen that even a very small depth of penetration will have a measurable effect on the value of the blue primary. This bar also illustrates that the depth of penetration greater than approximately $0.05 \mathrm{~mm}$ cannot be reliably measured with such a camera. However, the fact that dermal melanin $i s$ present can be detected. 


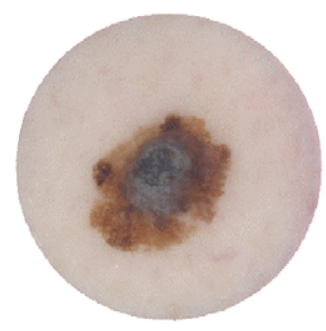

(a)

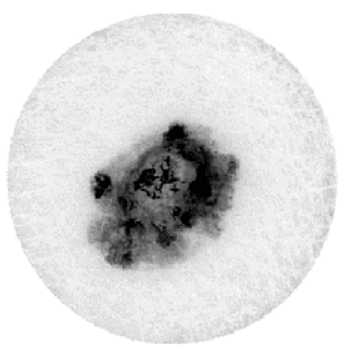

(b)

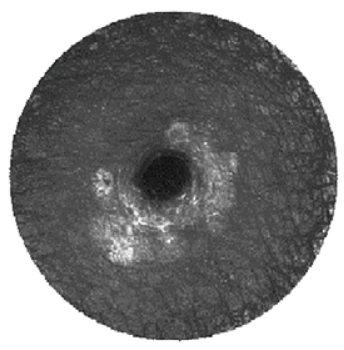

(d)

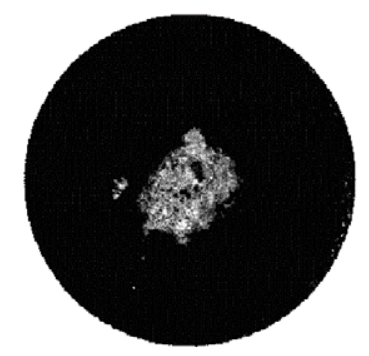

(c)

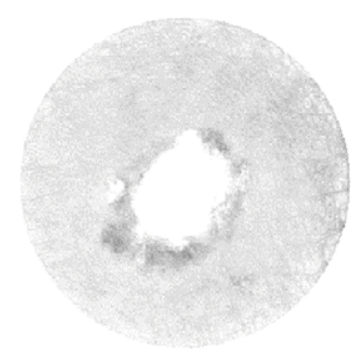

(e)

Fig. 6. (a) A colour image of a melanoma. Parametric maps showing (b) total melanin (darker=more); (c) dermal melanin (black=none, brighter = more); note that dermal melanin is present; (d) thickness of the papillary dermis (brighter=more); note the increased amount on the periphery and also a 'collagen hole' in the centre of the lesion where collagen was displaced by melanin; (e) blood (darker=more); note the absence of blood in the centre of the lesion-white area-and increased amount of blood on a periphery - an erythematous reaction. See the body of the paper for further explanations and comments.

several deep nests can be seen, including one near the periphery where, at the same location, a dark nodule can be seen in the total melanin map. The blood maps shows a very characteristic pattern associated with melanoma. The centre of the lesion is totally deprived of blood and this 'blood displacement' is co-located with the region of dermal melanin. This area of the lesion does not receive any nutrients and may turn necrotic. The periphery of the lesion shows increased blood supply. Note that this erythematous reaction cannot be seen in the original colour image because of the masking effect of the epidermal melanin which is present in increased quantities as can be seen in the total melanin map. The peripheral region in the collagen map shows the increased thickness-the fibrosis. These three signs together: increased epidermal melanin, erythematous reaction and fibrosis are strongly suggestive of the fact that the active cancer growth is occurring in this area. In the centre of the lesion, the presence of dermal melanin, the lack of blood and total lack of collagen (the so-called 'collagen hole', clearly seen in the collagen map) strongly suggest that melanin has totally replaced normal skin tissue. The fact that the hole is black indicates that the cancer has penetrated as far as the reticular dermis because no light is reflected from this region.

Fig. 7 shows a pale and fairly 'innocent' looking lesion, however the combination of increased epidermal melanin, the presence of dermal melanin, erythematous reaction and slight fibrosis suggest the presence of cancer, which was subsequently confirmed by histological examination.

\section{Model validation}

The objective of validation was to establish whether the model of colouration and the method of computing histological quantities from image colours are correct.

In the early stages of the development of the technique a simple experiment was carried out in which the locus of the skin colours generated by the model of colouration was compared with the distribution of colours obtained from images of the normal skin. Colour measurements acquired from the forearms of fifty two volunteers of various racial 


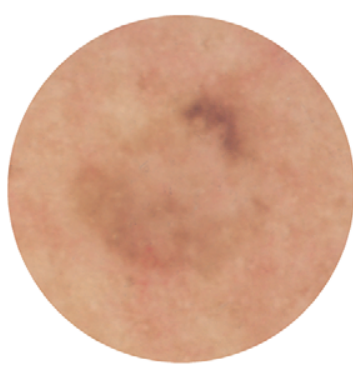

(a)

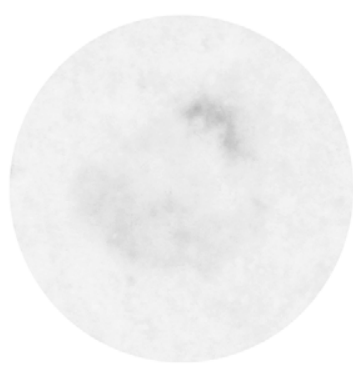

(b)

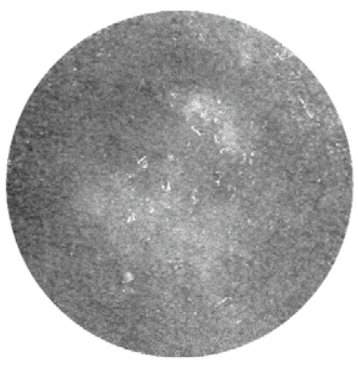

(d)

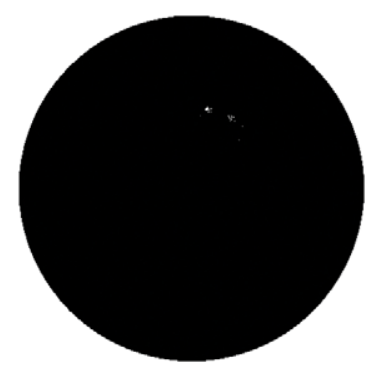

(c)

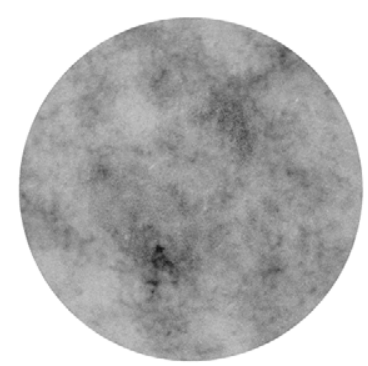

(e)

Fig. 7. (a) A colour image of an early melanoma (in situ). Parametric maps showing (b) total melanin (darker $=$ more); (c) dermal melanin (black=none, brighter =more); small speckles of dermal melanin are at 1 o'clock; (d) thickness of the papillary dermis (brighter $=$ more); note the increased amount in all areas of increased epidermal melanin; (e) blood (darker =more); note an erythematous reaction in the area corresponding to dermal melanin.

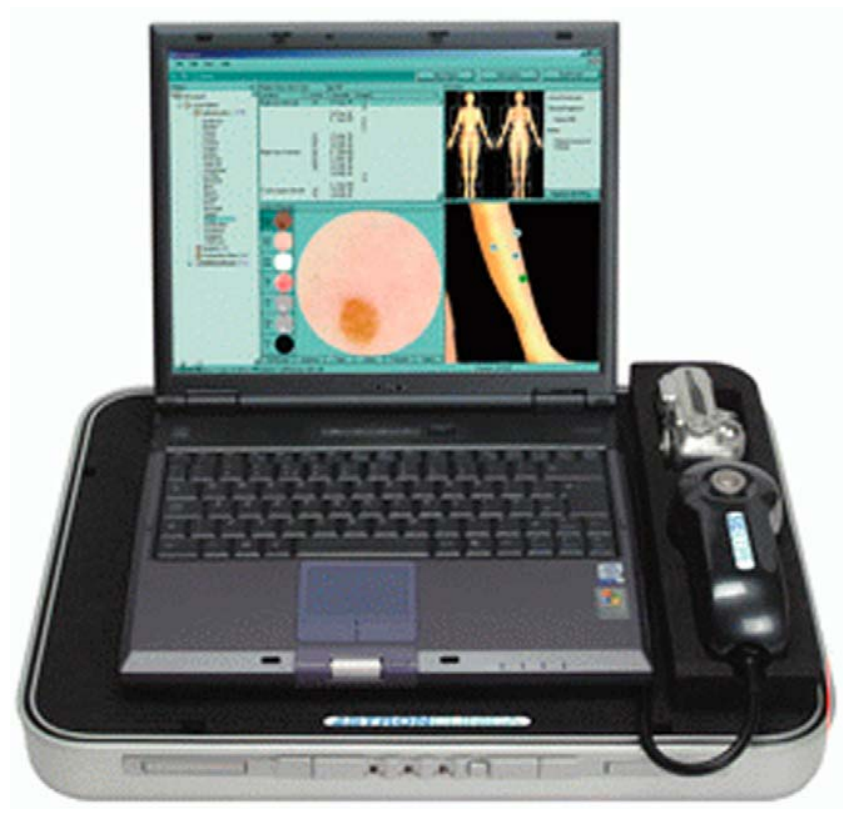

Fig. 8. SIAscope II-a certified commercial device developed using the model of skin colouration and the technique described in this paper (www.siascope.com). (This figure is available in colour, see the on-line version). origins were used for comparison with the model. According to standard experimental error analysis (Lyons, 1991) a model is valid if $66 \%$ of the measured data lies within one standard deviation. As $78 \%$ of the measured data was within one standard deviation from the theoretically predicted locus (Cotton and Claridge, 1996), we were satisfied that, in general, the data fits the model within the bounds of experimental error.

A more detailed recent study attempted to validate whether the magnitudes displayed in parametric images of total melanin, dermal melanin, blood and papillary dermis correspond to the magnitudes present in the skin. This proved to be a hard problem because it is extremely difficult to measure these parameters in vivo in the human skin. Thus it was decided only to carry out indirect comparisons at this stage (Moncrieff, 2001).

All the parametric maps used in the study were obtained using a SIAscope-a commercial, clinically approved device, operating according to the principles of the imaging method described above. Images of the skin are taken over an area of $24 \times 24 \mathrm{~mm}$, with spatial resolution of 0.04 $\mathrm{mm}$ per pixel. A number of spectrally filtered images are acquired in the range between approximately 400 and 1000 


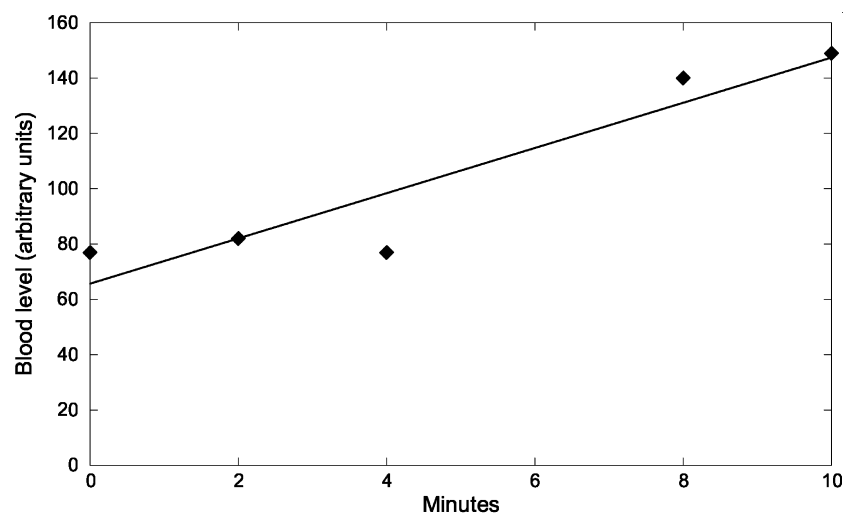

Fig. 9. The rhomboids show blood level as measured by a SIAscope during a vigorous exercise on an exercise bike at rest ( 0 minutes) and then at 2 minute intervals. The measurements represent the average brightness of a parametric map for the blood from a $24 \times 24 \mathrm{~mm}$ area of the skin. The line is a least squares fit through the points and it shows the increase in the blood level (line gradient $=8.2$ ).

$\mathrm{nm}$, calibrated and processed to compute the parametric maps. Fig. 8 shows SIAscope II.

For validation of the total melanin and blood levels the quantities in parametric maps were correlated with measurements from a Mexameter-an industry standard spectrometer designed to measure melanin and haemoglobin contents in the skin (CK Electronic, Cologne, Germany). 78 healthy volunteers took part in the study. Melanin measurements correlated almost perfectly $\left(R^{2}=0.905, p<\right.$ 0.0001 ), whereas for blood the $R^{2}$ values indicated only moderate correlation $\left(R^{2}=0.22, p<0.0001\right)$. These disappointing results for blood could be partially explained by the fact that the measurements were carried out only at the bottom fifth $(20 \%)$ of the working ranges of both instruments where sensitivity is at its lowest. The reason why only this low range was covered was that all the images were acquired at rest when the blood flow is minimal. In contrast, the melanin measurements covered over $60 \%$ of the instruments ranges due to a large range of skin colours captured from volunteers of different racial origins. Another source of weak correlation for blood is associated with the measurement method employed by Mexameter which does not take into account the fact that amount of light absorbed by haemoglobin is modulated by the thickness of collagen. In contrast, parametric maps for blood incorporate this factor and hence are likely to reflect real blood levels more accurately than Mexameter. A second set of experiments was therefore carried out to see whether parametric maps for blood reflect relative changes occurring in the skin during normal physiological processes. SIAscope measurements were taken from eight volunteers during vigorous exercise on an exercise bike at 2 minute intervals. The hypothesis was that the exercise would produce vasodilatation of the skin and the increase in blood supply would result in the increase of values in blood parametric maps. The results confirmed this hypothesis (see Fig. 9), although the small size of the data pool precluded more formal statistical analysis.

Validation of collagen maps was carried out by comparing them with histological cross-sections. On histological slides the collagen boundary is clearly visible and with appropriate scaling it should be theoretically possible to directly correlate the shape of this boundary with collagen thickness shown in a parametric map. However, due to the shrinking and warping of a specimen during the normal process of histological preparation, it is virtually impossible to correlate the exact point on a parametric map with the exact point in the slide. Thus validation was carried out visually by an experienced clinician comparing the presence/absence of major capillary ridges and, if present, their relative magnitudes. The study has shown that all the gross features measuring more than $100 \mu \mathrm{m}$ across were represented correctly in parametric maps of the collagen (Moncrieff, 2001).

\section{Clinical evaluation}

The method was evaluated in a clinical study at the Addenbrooke's Hospital, Cambridge, and at the Norwich Hospital. The objective of the study was to see whether the histological features which can be directly observed in parametric maps improve diagnosis of melanoma in comparison to standard clinical examination. Full details of the

Table 2

Diagnostic features associated with the parametric maps, and sensitivity and specificity of the individual features in melanoma classification

\begin{tabular}{|c|c|c|c|}
\hline Diagnostic feature & Parametric map & Sensitivity (\%) & Specificity (\%) \\
\hline Presence of dermal melanin & Dermal melanin & 96.2 & 56.8 \\
\hline $\begin{array}{l}\text { Areas within the lesion with no blood } \\
\text { present ('blood displacement') }\end{array}$ & Dermal blood & 75.0 & 70.3 \\
\hline $\begin{array}{l}\text { Increase in blood level on the lesion } \\
\text { periphery ('erythematous blush') }\end{array}$ & Dermal blood & 75.0 & 65.5 \\
\hline $\begin{array}{l}\text { Areas within the lesion with no collagen } \\
\text { present ('collagen holes') }\end{array}$ & Collagen thickness & 78.8 & 74.0 \\
\hline Asymmetry & Total melanin & 76.9 & 62.2 \\
\hline
\end{tabular}




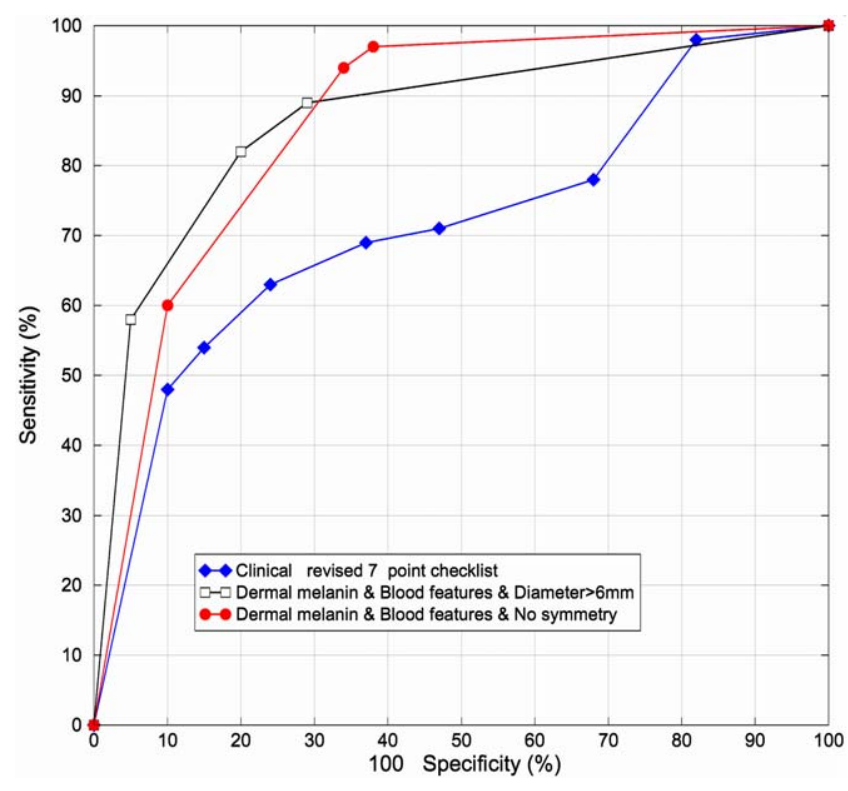

Fig. 10. Receiver-operator characteristic (ROC) curves for combinations of features, compared with clinical assessment and dermatoscopy. (This figure is available in colour, see the on-line version).

evaluation have been published earlier in a clinical journal (Moncrieff et al., 2002); this section provides a summary of these findings.

A set of 348 lesion images was collected in specialized dermatology clinics in Cambridge and Norwich using a SIAscope. Lesions were scored according to a revised seven-point checklist (Morton and MacKie, 1998)-a standard method in clinical assessment of lesions. Each imaged lesion was then excised and sent for histopathological analysis. Histology reports (melanoma versus nonmelanoma) were taken to be the ground truth. The set included 52 melanomas of various sub-types and at various stages of development, 28 of which has a Breslow thickness of $1 \mathrm{~mm}$ or less. Most of the non-melanomas were benign naevi. Factual and clinical information was also recorded, including gender, location on the body, diameter, symmetry and others.

In a blind study the parametric maps of the lesions, together with their clinical information and colour images, were examined visually by an experienced clinician. This preliminary analysis (Moncrieff et al., 2000) identified the features listed in Table 2 as being most strongly associated with melanoma. The table lists also sensitivity and specificity of the individual features.

The subsequent logistic regression analysis identified the combinations of the features which result in the best overall classification of melanoma (Moncrieff et al., 2002). Fig. 10 shows ROC curves for the two best combinations which were:

(1) The presence of dermal melanin, blood displacement and peripheral blush occurring together and no biaxial symmetry in dermal melanin (features from the parametric maps only).

(2) The presence of dermal melanin, blood displacement and peripheral blush occurring together and lesion diameter $>6 \mathrm{~mm}$ (parametric map features and clinical features).

The second combination of features is slightly better than the first, having $80.1 \%$ sensitivity and $82.7 \%$ specificity. For comparison the graph also shows the ROC curve for a clinical diagnosis based on a revised sevenpoint checklist alone.

\section{Discussion}

The key contribution of this work is a method for generating parametric images - a new and unique representation of the skin histology. Computer image analysis techniques for the analysis of pigmented skin lesions usually aim to correlate the lesion's appearance with its diagnosis. Visual features are usually based on the published checklists (MacKie, 1985; Fitzpatrick et al., 1988), and include colour, pigmentation, pigment variation, border irregularity, asymmetry, size, etc. The methods differ in the way that the measurements of these features are derived from digital images and the way that they are correlated with diagnosis. However, many lack explanatory power and in most instances they act as 'black boxes' which take in the images and output either numerical parameters or a putative diagnosis. Systems of this nature are not well accepted by practicing clinicians.

Our approach is fundamentally different in two ways. First, it does not concentrate on image patterns and image features, but through a physics-based interpretation of image colours it makes explicit the underlying histology. Second, by generating images showing the relative magnitudes of the histological entities, the lesion appearance can be correlated with its structure, thus providing an explanation as to why various skin diseases manifest themselves through particular visual signs. The model of normal skin colouration is representative of all the normal skins, irrespective of racial origin, age or gender (Cotton and Claridge, 1996). The structure remains the same, and the only differences are in the magnitudes of the parameters specifying level of melanin, level of haemoglobin and thickness of papillary dermis.

The following example may help to highlight the difference between traditional approaches, which focus on image features, and our approach. A physician trained in dermatoscopy will be asked to detect the presence of a 'blue-white veil'. According to Menzies et al. (1996) "histopathologically this corresponds to superficial fibrosis with melanophages and or pigmented melanin cells in the papillary dermis". In parametric maps both these histologi- 
cal features will be directly and explicitly represented, the increased level of collagen (fibrosis) in the collagen map and the presence of melanin in the dermal melanin map. Indeed, the study of these maps in reference to the optical effect of collagen and melanin can explain clearly the origin of the colour seen in the lesion. Increase in collagen thickness causes more light to be back-scattered thus making the affected area appearing brighter. As scatter in collagen is stronger for shorter (blue) wavelengths, the remitted light will take slightly bluish hue. Thus the blue-white veil, which is hard to detect, is caused by the increase in collagen thickness. It can be clearly seen that the dermatoscopic feature is abstract and its association with diagnosis has to be learned, whereas the parametric images show the underlying histology.

Spectral and hyperspectral imaging methods (Wallace et al., 2000; Elbaum et al., 2001) provide more complete recording of the remitted light than is the case for the traditional imaging methods. In the past spectral methods could only deliver point measurements and their serious weakness was that it was difficult to observe spatial patterns in the imaged objects. The advent of hyperspectral imaging devices has removed this disadvantage and it is now possible to collect a discrete spectrum for each pixel in the image in a matter of tens of seconds. This new data has been used for classification of abnormalities in skin images (Elbaum et al., 2001); however, the manner of analysis is the same as for the traditional imaging methods. Patterns in the spectra have taken the place of patterns in the images, and similar statistical techniques are applied leading to similar 'black box' classification.

It is interesting to compare the results of lesion classification based on the parametric maps with other diagnostic methods. A well known survey of the clinical accuracy of the diagnosis of malignant melanoma by Morton and MacKie (1998), reports $91 \%$ sensitivity and $99 \%$ specificity for consultant dermatologists with more than 10 years of experience. The respective figures for registrars are $79 \%$ and $98 \%$. In dermatoscopy, $72 \%$ sensitivity and $78 \%$ specificity, reported by Binder et al. (1997), are typical. The results obtained with the use of parametric maps $(80.1 \%$ sensitivity and $82.7 \%$ specificity) have sensitivity similar to that of registrars, but specificity is less than achieved by clinical assessment. We attribute a relatively high number of false positives to the rigorous way in which our study was conducted, with the clinicians being instructed to ignore the overall appearance of the lesion and to score only the presence or absence of specific features in the parameteric maps (Moncrieff et al., 2002). Extension of the analysis of parametric maps, as described in Section 9, should deal with this issue. The results from the parametric maps are better than from dermatoscopy and this can be attributed to the fact that the parametric maps provide the clinician with information which can be easily understood and interpreted because it is directly related to histology. By comparison, features in dermato- scopic images are of a more abstract nature and their interpretation requires a long period of training (Binder et al., 1995).

\section{Conclusions and future work}

From a practical point of view the new image interpretation method has been shown to contribute to improved diagnosis of skin lesions. However, there are many scientific challenges ahead. Work is currently in progress to provide improved verification of the method. As direct measurement of levels of melanin or blood in vivo, so that they can be compared to the quantities shown in parametric maps, are not very practical we are seeking to verify our methods through other indirect or ex vivo methods. For instance, it is possible to use immunohistochemical techniques to look at blood vessel and melanin distribution in pathology specimens of lesions that have been serially sectioned. It should be then possible to produce a threedimensional reconstruction of their distribution and carry out quantitative estimation. A synthetic melanin analogue (melaneze) with similar optical properties to natural melanin can be injected in specific amounts and localities and then imaged.

The spatial resolution in the plane of the skin is currently $0.04 \mathrm{~mm}$ per pixel and as such is satisfactory for resolving macroscopic features such as gross papillary ridges. However, the spatial definition of structures decreases with the increased depth because of strong isotropic scatter by collagen. Depth of the descent of dermal melanin is one of the important factors affecting patient prognosis and the preferred treatment. Whereas the presence of melanin in the dermis can be detected with great sensitivity, the accuracy of its depth estimate decreases with the increase in depth. In malignant melanoma normally the greater the depth the greater the volume of melanin at a given location in the skin. Such increased volume will absorb proportionally greater amounts of light and reduce the amount of remitted light. To measure such low levels may require a camera with increased sensitivity, possibly with nitrogen cooling, which would lead to increased cost of the imaging equipment, typically by an order of magnitude.

This imaging method is not fully tomographic, i.e. it does not show properties of individual three-dimensional voxels, but only the characteristics of a cone of tissue below each image pixel. New experimental imaging methods such as optical coherence tomography (OCT) (e.g. Pagnoni et al., 1999) are making tomographic imaging of the skin possible and should lead to even better insights into the skin physiology, but again at greater expense.

At present the features in the parametric maps have to be identified and scored by trained medical staff. The key features used for diagnosis, the presence of dermal melanin and blood displacement with peripheral erythema, are easy 
to detect as confirmed by inter- and intra-observer reproducibility experiments (Moncrieff et al., 2002). It is possible that the lesion classification rates can be improved by introducing computer support. For example, a small area of blood deprivation of the size of 2-3 pixels can be missed by an observer, whereas it can be easily detected by a simple computer method (Satta and Claridge, 2002). Other important diagnostic features, such as for example the level of symmetry in the pattern of melanin distribution, are known to be difficult to assess visually (Hibbin, 2001). More-objective computer methods are likely to improve the consistency of scoring. Work is in progress on a number of aspects of computer-assisted detection and quantification of features in parametric maps, including the ones mentioned above.

Colour changes of many body tissues are used for diagnosing diseases. Most tissues have regular laminar structure, collagenous framework and contain a small number of pigments. The colour interpretation method presented in this paper can be clearly extended to other tissues (Preece and Claridge, 2002) and in some cases it may be an attractive alternative to biopsy or other invasive examinations.

\section{Acknowledgements}

This project is supported by the Engineering and Physical Sciences Research Council, UK, grant number GR/M53035. The authors gratefully acknowledge the referees' comments.

\section{References}

Aitken, J.F. et al., 1996. Reliability of computer image analysis of pigmented skin lesions of Australian adolescents. Cancer 78 (2), 252-257.

Anderson, R., Parrish, B.S., Parrish, J., 1981. The optics of human skin. J. Investigative Dermatol. 77 (1), 13-19.

Binder, M., Puespoeck-Schwarz, M., Steiner, A. et al., 1997. Epiluminescence microscopy of small pigmented skin lesions: short-term formal training improves the diagnostic performance of dermatologists. J. Am. Acad. Dermatol. 36, 197-202.

Binder, N. et al., 1995. Epiluminescence microscopy. A useful tool for the diagnosis of pigmented skin lesions for formally trained dermatologists. Arch. Dermatol 131, 286-291.

Bono, A., Tomatis, S. et al., 1996. Invisible colours of melanoma. A telespectrophotometric diagnostic approach on pigmented skin lesions. Eur. J. Cancer 32A, 727-729.

Breslow, R.A., 1970. Thickness, cross-sectional areas and depth of invasion in the prognosis of cutaneous melanoma. Ann. Surg. 172 (5), 902-908.

Cai, J., Goshtasby, A., 1999. Detecting human faces in color images. Image and Vision Computing 18, 63-75.

Claridge E., Preece S.J., 2003. An inverse method for the recovery of tissue parameters from colour images. In: Taylor, C., Noble, J.A. (Eds.), Information Processing in Medical Imaging. Springer, Berlin, LNCS2732, pp. 306-317.
Computerized Medical Imaging and Graphics 16(3) (1992). Special issue on Melanoma.

Cotton, S.D., Claridge, E., 1996. Developing a predictive model of human skin colouring. In: Vanmetter, R.L., Beutel, J. (Eds.), Proceedings of the SPIE Medical Imaging, Vol. 2708, pp. 814-825.

Egan, W.G., Hilgeman, T.W., 1979. In: Optical Properties of Inhomogeneous Materials. Academic Press, New York.

Elbaum, M., Kopf, A.W., Rabinovitz, H.S. et al., 2001. Automatic differentiation of melanoma from melanocytic naevi with multispectral digital dermoscopy: A feasibility study. J. Am. Acad. Dermatol. 44 (2), 207-218.

Ercal, F., Chawla, A., Stoecker, W. et al., 1994. Neural network diagnosis of malignant melanoma from color images. IEEE Trans. Biomed. Eng. $41,837-845$.

Fitzpatrick, T.B., Rhodes, A.R., Sober, A.J., Mihn, M.C., 1988. Primary malignant melanoma of the skin: the call for action to identify persons at risk, to discover precursor lesions, to detect early melanoma. Pigment Cell. 9, 110-117.

Ganster, H., Pinz, A., Kittler, H. et al., 1997. Computer aided recognition of pigmented skin lesions. Melanoma Res. 7, 19.

Ganster, H. et al., 2001. Automated melanoma recognition. IEEE Trans. Med. Imaging 20 (3), 233-239.

Greenspan, H., Goldberger, J., Eshet, I., 2001. Mixture model for facecolor modelling and segmentation. Pattern Recognition Lett. 22 (14), $1525-1536$.

Hibbin, A., 2001. Perception of Natural Symmetry: A Comparison of Human Vision and a Computer Model. MSc in Cognitive Science Thesis, Schools of Computer Science and Psychology, The University of Birmingham.

Huang, C.L., 1999. Border irregularity: atypical moles versus melanoma. Eur. J. Dermatol. 18 (2), 164-171.

Husemann, R. et al., 1997. In: Altmeyer, P., Hoffman, K., Stucker, M. (Eds.), Computerised Diagnosis of Skin Cancer Using Neural Networks. International Congress on Skin Cancer and UV Radiation, Bochum, Germany, pp. 1052-1063.

Jones, M.J., Regh, J.M., 2002. Statistical color models with application to skin detection. Int. J. Computer Vision 46, 81-96.

Kahofer, P. et al., 2002. Tissue counter analysis of dermatoscopic images of melanocytic skin tumours: preliminary findings. Melanoma Res. 12, $71-75$.

Lyons, L., 1991. In: A Practical Guide to Data Analysis for Physical Science Students. Cambridge University Press, Cambridge.

MacKie, R., 1985. In: An Illustrated Guide To the Recognition of Early Malignant Melanoma. University Department of Dermatology, Glasgow.

Menzies, S. et al., 1996. In: An Atlas of Surface Microscopy of Pigmented Skin Lesions. McGraw-Hill, New York.

Menzies, S., Crook, B., McCarthy, W. et al., 1997. Automated instrumentation and diagnosis of invasive melanoma. Melanoma Res. 7, 13.

Moncrieff, M., 2001. The Clinical Application of Spectrophotometric Intracutaneous Analysis for the Diagnosis of Cutaneous Malignant Melanoma. DM Thesis, School of Medicine, Health Policy and Practice, Faculty of Science, The University of East Anglia.

Moncrieff, M. et al., 2000. Spectrophotometric intracutaneous analysis assists in the identification of dermatoscopic features. J. Eur. Acad. Dermatol. Venerol. 14 (S1), 220.

Moncrieff, M., Cotton, S., Claridge, E., Hall, P.N., 2002. Spectrophotometric Intracutaneous analysis: a new technique for imaging pigmented skin lesions. Br. J. Dermatol. 146 (3), 448-457.

Morton, C.A., MacKie, R.M., 1998. Clinical accuracy of the diagnosis of cutaneous malignant melanoma. Br. J. Dermatol. 138, 283-287.

Pagnoni, A. et al., 1999. Optical coherence tomography in dermatology. Skin Res. Technol. 5, 83-87.

Preece, S.J., Claridge, E., 2002. Monte Carlo modelling of the spectral reflectance of the human eye. Phys. Med. Biol. 47, 2863-2877.

Satta, F., Claridge, E., 2002. Detection of blood deprived regions in SIAgraph images of pigmented skin lesions. In: Houston, A., 
Zwiggelaar, R. (Eds.), Medical Image Understanding and Analysis 2002 , pp. $57-60$.

Seidenari, S. et al., 1999. Digital videomicroscopy and image analysis with automatic classification for detection of thin melanomas. Melanoma Res. 9 (2), 163-171.

Spiegel, M.R., 1962. In: Tbeory and Practice of Advanced Calculus. McGraw-Hill, New York.

Tsumura, N. et al., 1999. Independent component analysis of skin color image. J. Opt. Soc. Am. A 16 (9), 2169-2176.
Tsumura, N. et al., 2001. Mapping pigmentation in human skin from a multi-channel visible spectrum image by inverse optical scattering technique. J. Imaging Sci. Technol. 45 (5), 444-450.

Wallace, V.P. et al., 2000. Spectrophotometric assessment of pigmented skin lesions: methods and feature selection for evaluation of diagnostic performance. Phys. Med. Biol. 45 (3), 735-751. 